\title{
3-D Poststack Migration Using the Adjoint Operator of Modeling
}

\author{
Qiqiang Yang
}

\author{
Department of Mathematics, Hainan tropical ocean College, Sanya, China \\ keeponmoving@126.com
}

\section{Keywords: 3-D, Migration, Adjoint operator, Modeling}

\begin{abstract}
From the modeling operator, we derive its adjoint operator which is used to migrate 3-D poststack data. It forms a theoretical foundation for the reverse-time migration. The method would be helpful to get a new way to improve the result of conventional reverse-time migration. The inference of the adjoint operator is adaptive to other modeling method including both poststak modeling and prestack modeling. In this paper, we focus on poststack migration for 3-D problems. And the Fourier method for time stepping is discussed. Furthermore, we adopt the numerical software FFTW to perform Fourier transformation, with which the efficiency of the algorithm is greatly improved.
\end{abstract}

\section{Introduction}

Through the increasing availability of large-scale computing resources, three-dimensional (3-D) seismic modeling and migration [1,2] are now feasible. The 3-D Fourier method for one-way wave extrapolation presented in this paper is a generalization of the 2-D Fourier method [3] except that FFTW is used rather than general fast Fourier transforms (FFT). FFTW's performance is typically superior to that of other publicly available FFT software, and is even competitive with vendor-tuned codes [4].

Logically, the adjoint is the first step and a part of all subsequent steps in this process. Surprisingly, in practice the adjoint sometimes does a better job than the inverse [5]. This is because the adjoint operator tolerates imperfections in the data and does not demand that the data provide full information. Sometimes the adjoint operator is called the "back projection" operator because information propagated from earth to data is projected backward. The adjoint operator is often defined as the transpose of the matrix in mathematics, and in seismic data processing, it has some differences. The paper discusses and deduces the adjoint of poststack modeling operator. The proposed migration method using the adjoint of modeling operator is a type of depth migration in which the observations are extrapolated in time rather than in depth. It has two basic forms, one for stacked sections, in which the imaging conditions is implicit at time zero, and one for prestack common-source gathers, in which the imaging conditions is explicit and each point in the image space has its own image time.

The author expounds the essence of three dimensional poststack migration from the perspective of adjoint operator using exploding-reflector data. First we give a review of the Fourier modeling method. Next, the adjoint operator of 3-D modeling operator is deduced. Finally, the results are shown.

\section{3-D wave extrapolation using up-ward wave equation}

When density is constant, the acoustic wave equation reads

$$
\frac{\partial^{2} p}{\partial x^{2}}+\frac{\partial^{2} p}{\partial y^{2}}+\frac{\partial^{2} p}{\partial z^{2}}=\frac{1}{v^{2}} \frac{\partial^{2} p}{\partial t^{2}}
$$

where $p=p(x, y, z, t)$ represents the pressure, $v(x, y, z)$ is the velocity.

The Fourier transform have the differential property:

$$
F[d f(x) / d x]=i \omega F[f(x)], \quad F\left[d^{2} f(t) / d t^{2}\right]=-\omega^{2} F[f(t)],
$$

where $F(t)$ and $F^{-1}(t)$ denote the performance of a Fourier transform and a reverse Fourier transform 
to $t$, respectively. With the second order differential property, we get $k_{x}^{2}+k_{y}^{2}+k_{z}^{2}=\omega^{2} / v^{2}$. The upcoming waves are characterized by the dispersion equation $\omega=v k_{z}\left[k_{x}^{2}+k_{y}^{2}+k_{z}^{2}\right]^{1 / 2}$, which governs the interdependence between the temporal frequency $\omega$ and the wavenumbers or spatial frequencies $k_{x}, k_{y}, k_{z}$. With the first order differential property, we have

$$
F\left[\frac{\partial P\left(k_{x}, k_{y}, k_{z}, t\right)}{\partial t}\right]=i \omega F\left[P\left(k_{x}, k_{y}, k_{z}, \omega\right)\right]=i v k_{z}\left[1+\left(k_{x} / k_{z}\right)^{2}+\left(k_{y} / k_{z}\right)^{2}\right]^{1 / 2} F\left[P\left(k_{x}, k_{y}, k_{z}, t\right)\right]
$$

For a homogeneous medium in which $v$ is constant, we can immediately write the differential equation which describes upward propagation waves as

$$
\frac{\partial P\left(k_{x}, k_{y}, k_{z}, t\right)}{\partial t}=i v k_{z}\left[1+\left(k_{x} / k_{z}\right)^{2}+\left(k_{y} / k_{z}\right)^{2}\right]^{1 / 2} P\left(k_{x}, k_{y}, k_{z}, t\right) \text {. }
$$

The solution of Eq. 4 is $P\left(k_{x}, k_{y}, k_{z}, t+\Delta t\right)=P\left(k_{x}, k_{y}, k_{z}, t\right) e^{i v k_{z}\left[1+\left(k_{x} / k_{z}\right)^{2}+\left(k_{y} / k_{z}\right)^{2}\right]^{1 / 2} d t}$. If velocity has spatial variations, we express Eq.3 for the wave field $p(x, y, z, t)$, defined in the $(x, y, z)$ domain as

$$
\frac{\partial p(x, y, z, t)}{\partial t}=v F_{3}^{-1}\left\{i k_{z}\left[1+\left(k_{x} / k_{z}\right)^{2}\right]^{1 / 2} F_{3}(p)\right\},
$$

where $F_{3}$ and $F_{3}^{-1}$ are operators representing the triple Fourier transform from the $(x, y, z)$ domain to the $\left(k_{x}, k_{y}, k_{z}\right)$ domain and its inverse. Eq.5 cannot be written without the transform operator $F_{3}$ and $F_{3}^{-1}$, since the multiplication of $P\left(k_{x}, k_{y}, k_{z}, t\right)$ by $i v k_{z}\left[1+\left(k_{x} / k_{z}\right)^{2}+\left(k_{y} / k_{z}\right)^{2}\right]^{1 / 2}$ cannot be expressed in the $(x, y, z)$ space. In the numerical solution method for Eq.4, we employ the time differencing scheme

$$
p(t+\Delta t) \approx p(t)+\frac{\partial p(t)}{\partial t} \Delta t+\frac{\partial^{2} p(t)}{\partial t^{2}} \frac{\Delta t^{2}}{2}+\frac{\partial^{3} p(t)}{\partial t^{3}} \frac{\Delta t^{3}}{3 !},
$$

which states that the wave field at $t+\Delta t$ can be approximated from the wave field and its derivatives at $t$. Given $p(x, y, z, t)$ from the previous time step or from the initial conditions, $\partial p / \partial t$ is obtained by following the computational step outlined above for Eq.5: :

$$
\frac{\partial^{2} p}{\partial t^{2}}=v F_{3}^{-1}\left\{i k_{z}\left[1+\left(\frac{k_{x}}{k_{z}}\right)^{2}+\left(\frac{k_{y}}{k_{z}}\right)^{2}\right]^{1 / 2} F_{3}\left(\frac{\partial p}{\partial t}\right)\right\}, \frac{\partial^{3} p}{\partial t^{3}}=v F_{3}^{-1}\left\{i k_{z}\left[1+\left(\frac{k_{x}}{k_{z}}\right)^{2}+\left(\frac{k_{y}}{k_{z}}\right)^{2}\right]^{1 / 2} F_{3}\left(\frac{\partial^{2} p}{\partial t^{2}}\right\} .\right.
$$

\section{3-D migration using the adjoint of the modeling operator}

The process of modeling can be expressed as follows, wave fields are extrapolated to the next time step according to Eq.6, next the values in the recording positions are extracted to put into data record, and then the above operations are repeated. We symbolically denote the process expressed in Eq.6 as operator $L$. Eq.6 can be written as $\boldsymbol{p}_{\boldsymbol{i + 1}}=\boldsymbol{L} \boldsymbol{p}_{\boldsymbol{i}}$, where $p_{i}$ denotes $p(x, y, z, i \Delta t)$. The wave field $p_{i}$ is considered to be a $n z \times n x$ matrix with each item a vector of length $n y$ and the total number of carried time steps is $n t$, the data record is considered as a $n t \times n x$ matrix with each item a vector of length ny. The operation of extracting values of wav field at each recording point is expressed by $A_{i+1}=C_{i+1} p_{i+1}$,where $A_{i+1}$ represents the matrix whose rows are padded with zero vector except that the $(\boldsymbol{i}+\mathbf{1})$ th row has the same items as $A, C_{i+1}$ is a $n t \times n z$ matrix:

$$
C_{i+1}=\left[\begin{array}{c}
0,0, \ldots, 0 \\
0,0, \ldots, 0 \\
\ldots \ldots \ldots . . \\
1,0, \ldots, 0 \\
\ldots \ldots \ldots . . \\
0,0, \ldots, 0
\end{array}\right]
$$

the only nonzero element unity is located at the $(\boldsymbol{i}+\mathbf{1})$ th row and the first column. The record 
matrix can be written as

$$
A=\sum_{i=1}^{n t} A_{i}=\sum_{i=1}^{n t} C_{i} p_{i}=\sum_{i=1}^{n t} C_{i} L^{i} p_{0},
$$

where $L^{i}$ represents the process of time stepping for $i$ times according to Eq.6. The adjoint operation of Eq.9 is

$$
\tilde{p}_{0}=\left(\sum_{i=1}^{n t} C_{i} L^{i}\right)^{\prime} A=\sum_{i=1}^{n t} L^{i^{\prime}} C_{i}^{\prime} \tilde{A}=\sum_{i=1}^{n t}\left(L^{\prime}\right)^{i} C_{i}^{\prime} \tilde{A}
$$

where $C_{i}^{\prime}$ is the adjoint operator of $C_{i}$, i.e. the transpose of $C_{i}, L^{\prime}$ is the adjoint operator of $L$ and represents the reverse-time stepping of wave field expressed as

$$
p(t-\Delta t) \approx p(t)-\frac{\partial p(t)}{\partial t} \Delta t+\frac{\partial^{2} p(t)}{\partial t^{2}} \frac{\Delta t^{2}}{2}-\frac{\partial^{3} p(t)}{\partial t^{3}} \frac{\Delta t^{3}}{3 !},,
$$

$\left(L^{\prime}\right)^{i}=L^{i}$ represents $i$ times of reverse-time stepping according to Eq.13. Let $\widetilde{B}_{i}=C_{i}^{\prime} A_{i}$, we describe Eq.10 as follows

$$
\begin{aligned}
& \tilde{P}_{0}=\sum_{i=1}^{n t}\left(L^{\prime}\right)^{i} \tilde{B}_{i}=\left(L^{\prime}\right)^{n t-1} L^{\prime} \tilde{B}_{n t}+\left(L^{\prime}\right)^{n t-1} \tilde{B}_{n t-1}+\sum_{i=n t-2}^{1}\left(L^{\prime}\right)^{i} \tilde{B}_{i} \\
= & \left(L^{\prime}\right)^{n t-1}\left(L^{\prime} \tilde{B}_{n t}+\tilde{B}_{n t-1}\right)+\left(L^{\prime}\right)^{n t-2} \tilde{B}_{n t-2}+\sum_{i=n t-3}^{1}\left(L^{\prime}\right)^{i} \tilde{B}_{i}=\left(L^{\prime}\right)^{n t-1}\left(L^{\prime} \tilde{B}_{n t}+\tilde{B}_{n t-1}\right)+\left(L^{\prime}\right)^{n t-2} \tilde{B}_{n t-2}+\sum_{i=n t-3}^{1}\left(L^{\prime}\right)^{i} \tilde{B}_{i} \\
= & \left.\left(L^{\prime}\right)^{n t-2}\left(L^{\prime}\left(L^{\prime} \tilde{B}_{n t}+\tilde{B}_{n t-1}\right)\right)+\tilde{B}_{n t-2}\right)+\left(L^{\prime}\right)^{n t-3} \tilde{B}_{n t-3} \sum_{i=n t-4}^{1}\left(L^{\prime}\right)^{i} \tilde{B}_{i} \\
= & \ldots \ldots . \\
= & L^{\prime}\left(L^{\prime}\left(\ldots\left(L^{\prime}\left(L^{\prime} \tilde{B}_{n t}+\widetilde{B}_{n t-1}\right)+\tilde{B}_{n t-2}\right)+\ldots\right)+\tilde{B}_{1}\right)
\end{aligned}
$$

It is obvious that

$$
\widetilde{B}_{i}=C_{i}^{\prime} A_{i}=\left[\begin{array}{l}
a_{i, 1}, a_{i, 2}, \ldots, a_{i, n x} \\
0,0, \ldots, \ldots \ldots \ldots . . . \\
\ldots \ldots \ldots \ldots \ldots . . . \\
0,0, \ldots, \ldots \ldots \ldots . . .
\end{array}\right],
$$

where $a_{i, 1}, a_{i, 2}, \ldots, a_{i, n x}$ are the values of the first row matrix $\widetilde{B}_{i}$.

The process of migration using the adjoint operator of modeling operator transfers data from the $(x, y, t)$ plane to the $(x, y, z)$ plane as follows. Each time step during migration consists of two operations: the wave equation carries all the previous energy away from the upper boundary of the $x-y-z$ plane toward its migrated position; then, values extracted from the seismograms profile along that slice of constant time that corresponds to the current iteration are inserted at each recording point.

\section{Numerical example}

A three dimensional velocity model with a flat reflector and a syncline shown in Fig.1.a-c is used to test the method. This is a complicated example both in the shape of reflector and in the velocity distribution. The seismic time section (Fig. 1d-f) was migrated to produce the image in the $x-y-z$ plane (Fig. 1g-i) with the method using the adjoint of modeling operator. A Fourier finite-difference migrated result is also shown in Fig. $1 \mathrm{j}-\mathrm{l}$ as a comparison to Fig. 1g-i. It is clear that the migrated result using the adjoint operator of modeling operator is globally better than that using Fourier finite-difference migration method. 


\section{Summary}

Migration using the adjoint of modeling operator can be performed as the solution of a boundary value problem in which the data are extrapolated backward in time. The process is the same as the reverse-time migration. Thus, the method gives a new viewpoint of the reverse-time migration and casts lights on how to improve the effect of reverse time migration since the adjoint operator is frequently used in optimization problem.

\section{Acknowledgements}

This research was supported by Hainan Natural Science Foundation (Grant No. 113008).

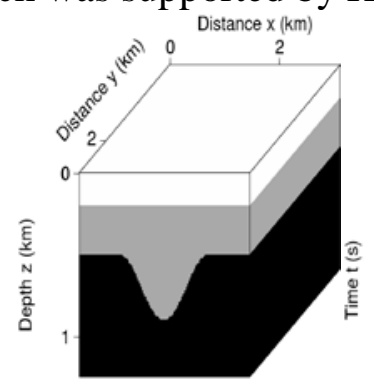

(a)

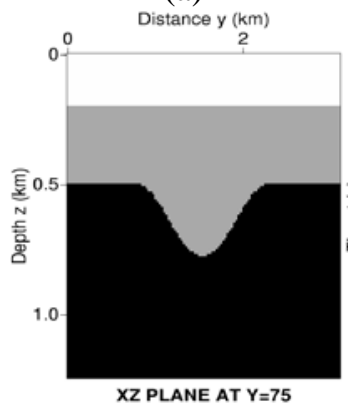

(b)

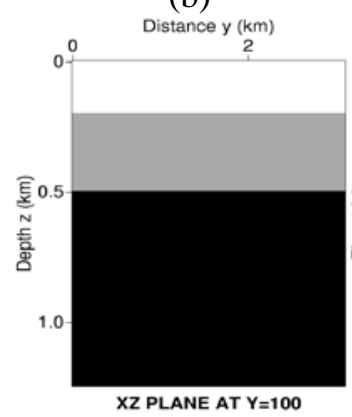

(c)

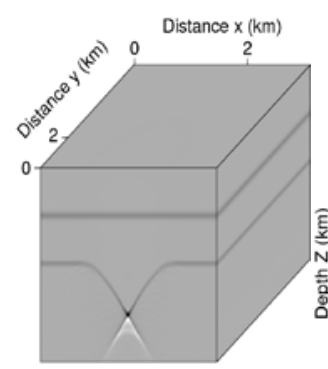

(d)

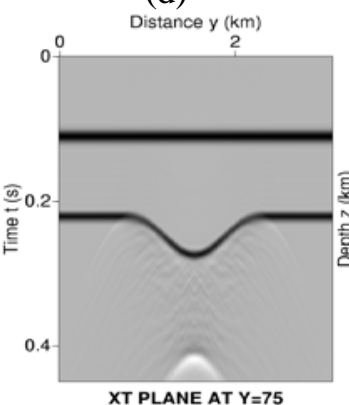

(e)

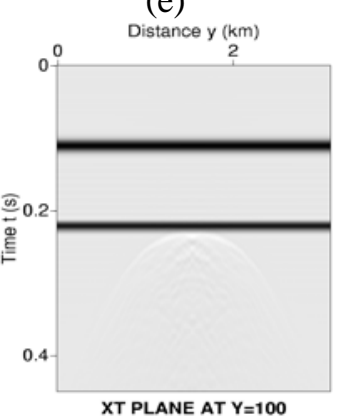

(f)

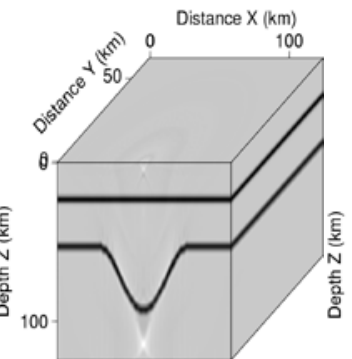

(g)

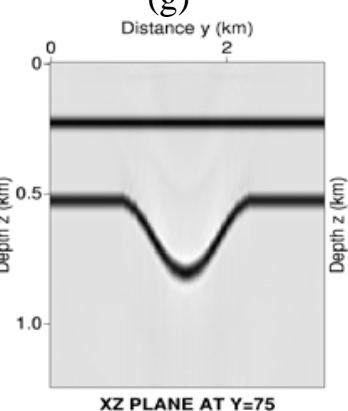

(h)

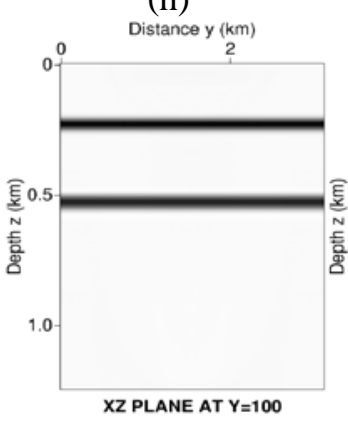

(i)

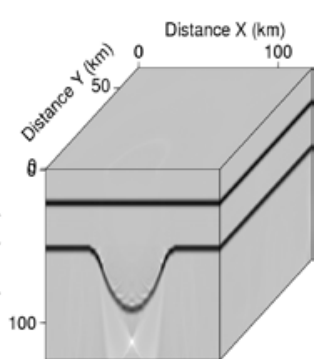

(j)

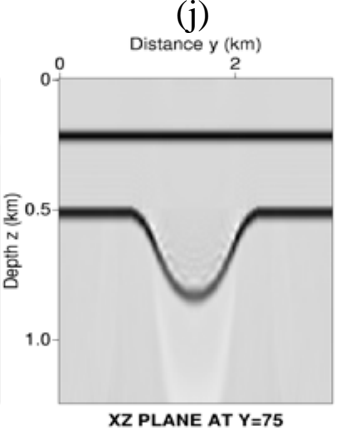

(k)

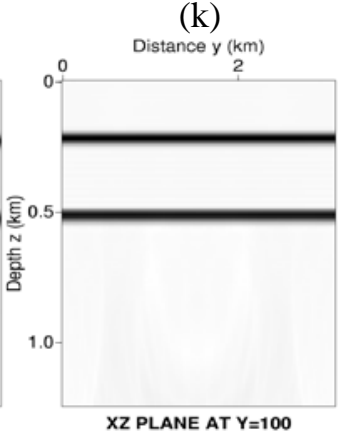

(l)

Fig.1. A numerical example for 3-D synthetic data.

\section{References}

[1] Yang, Q.Q., 3-D acoustic modeling by a Hartley method, Journal of Applied Geophysics, 70 (20 10), $169-180$.

[2] Yang, Q. Q. , Zhang,S. L, 3-D synthesized areal shot-record residual migration velocity analysis, Journal of Geophysics and Engineering, 6 (2009), 35-42.

[3] Gazdag, J., Modeling of acoustic wave equation by transform methods, Geophysics, 46 (1981), 854-859.

[4] http://www.fftw.org/.

[5] Kreiss, H. O., and Oliger, J., Comparision of accurate methods for the integration of hyperbolic equations. Tellus, 24 (1972), 199 255. 